Research Journal of Biological Sciences 7 (2): 92-96, 2012

ISSN: $1815-8846$

(C) Medwell Journals, 2012

\title{
Ability to Determine the Prognostic Value of Plasma Selenium Levels in the Diagnosis of Preeclampsia
}

\author{
${ }^{1}$ Sedighe Forouhari, ${ }^{5} \mathrm{Z}$ ahra Ghaemi, ${ }^{2}$ Mohammad Hosein Dabbaghmanesh, \\ ${ }^{1}$ Faride Vaziri, ${ }^{3}$ Zohreh Tavana and ${ }^{4}$ Mehrab Sayyadi \\ ${ }^{1}$ Faculty of Nursing and Midwifery, ${ }^{2}$ Endocrine and Metabolism Research Center, \\ ${ }^{3}$ Department of Obstetrics and Gynecology, ${ }^{4}$ Department of Statistics, \\ Shiraz University of Medical Sciences, Shiraz, Iran \\ ${ }^{5}$ Department of Midwifery, Estahban Azad University, Estahban, Iran
}

\begin{abstract}
Preeclampsia, a pregnancy-related condition that its specific etiology still remains obscure is recognized as the leading cause of morbidity and mortality in mothers and babies. Considering this, finding an index for predicting and preventing it seems vital. This study was designed by considering the theory of oxidative stress in the pathogenesis of preeclampsia and the role of selenium as an antioxidant in the prevention and detection of preeclampsia and the aim of determining the prognostic value of plasma selenium levels in the diagnosis of preeclampsia. In a nested case control study, a random sample of 600 normal primigravida in their 1st 24-28 weeks by screening participated in the study and the first blood sampling was performed on them. These blood samples after centrifugation for separating plasma were frozen in special freezers at temperatures of $70^{\circ} \mathrm{C}$. After 3 months follow-up of all subjects, 39 of the participants developed preeclampsia. They formed a case group and second phase of blood sampling was performed on them. Among remaining subjects those that till $48 \mathrm{~h}$ after gestation were healthy with matching gestational age and receiving supplemental in a systematic method formed the controls and the second phase of blood sampling was performed. After delivery of all 600 pregnant, the amount of selenium in blood samples of first and second case and control groups was measured. Calculated odds ratio showed that the odds for people with selenium $<62.2$ for being affected by preeclampsia were 9.52 times more compared with people with average selenium. Accordingly, the best cut point was estimated $66.1 \mu \mathrm{g} \mathrm{L}{ }^{-1}$ with sensitivity of $57.9 \%$ and specificity of 90 .
\end{abstract}

$\underline{\text { Key words: } \text { Preeclampsia, plasma selenium levels, primigravida, eltiology, morbidity, pathogenesis }}$

\section{INTRODUCTION}

Attention to maternal health is one of the pillars of any society because attention to the health of mothers is directly related to the health of other family members (Mehri, 2003). Hypertension disorders which complicate the pregnancy are common disorders that along with hemorrhage and infection form a deadly triad and are the main cause of pregnancy morbidity and mortality. Since, preeclampsia is one of the leading causes of maternal and neonatal mortality in the whole world since its discovery in the 19th century, prevention of preeclampsia has always been considered by scholars. Although, it has been studied extensively yet its incidence and intensification in pregnancy have not been resolved and hypertension disorders have remained the most important unsolved problems in obstetrics and gynecology. It is said that preeclampsia occur in $5 \%$ of all pregnancies but there is considerable diversity in this field. Preeclampsia is an unknown disease with several conjectures and theories. Despite identification of many risk factors in occurrence of preeclampsia, its specific etiology remains obscure (Hacker and Moor, 2004). Researchers have attempted to identify early markers of dysfunction of endothelial cells (Cunningham and Williams, 2005; Hacker and Moor, 2004; Scott et al., 2005).

Redman et al. (1999) predicted that such dysfunction of endothelial cells in preeclampsia may arise from generalized disturbance of maternal systemic inflammatory response in pregnancy. These findings about the effects of oxidative stress in preeclampsia have increased interest and attention to the potentially beneficial effects of antioxidants in the prevention of preeclampsia (Zhang et al., 2002). Antioxidants are a diverse group of compounds that act in order to prevent excessive production of toxic free radicals and the resulted damages (Cunningham and Williams, 2005). Selenium as

Corresponding Author: Zahra Ghaemi, Department of Midwifery, Estahban Azad University, Estahban, Iran 
a trace element in the structure of some antioxidants such as glutathione peroxidase, inhibit lipid peroxidase (Shils et al., 2006). Conflicting findings in relation to selenium concentrations in preeclamptic women has been reported. As some studies have pointed to reduced concentrations of selenium in plasma or serum (Kosanovic et al., 2002) and others to increase selenium (in plasma or leukocyte) in preeclamptic than normal pregnant women (Gromadzinska et al., 1998; Mahomed et al., 2000). Since the amount of selenium in different bodies due to the plant, soil, chemical regions and rainfall situation is different, sometimes contradictory results in different parts of the world's were obtained (Margaret et al., 2003). Measuring selenium levels using biochemical methods from samples obtained from hair, nails, blood and urine may be possible (Shils et al., 2006). Since at present there is no valid, reliable and economical screening test for early detection of preeclampsia (Meads et al., 2008) and on the other hand in recent years, the maternal care program the aims of improving the quality of maternal care, pre and post nata to reduce mortality rates in particular is one of the programs of the Department of Health, a valuable attempt to provide, maintain and improve maternal health in society becomes essential.

\section{MATERIALS AND METHODS}

This study was a nested case-control study and is designed to investigate the prognostic value of plasma selenium levels in the diagnosis of preeclampsia in Educational, Therapeutic Centers of Shiraz. The sample size according to the purposes of the project, type and method of the study and based on the previous studies with the size of $d=0.06$, variance of $\delta=0.09$, power of $80 \%$ and reliability of $95 \%$ was 39 individuals in each group. Regarding the type of the study it was predicted that in order to get the required minimum sample size in the case group, at least 650 individuals in the 1 st phase must participate. Therefore with regard to the study inclusion criteria: singleton pregnancy-gestational age between 24-28 weeks based on first trimester ultrasound exam, no history of chronic hypertension, no use of selenium-containing multivitamins during the past year, living in Shiraz during the last 2 years, no history of recognized internal diseases during pregnancy such as: kidney disease, diabetes, thyroid, 650 pregnant women participated in the study and finally shown the exclusion criteria of the study such as incidence of preterm delivery before the second phase of the test and occurrence of any internal diseases at each stage of sampling, 48 were excluded from this study population.

In order to obtain necessary information, a designed questionnaire by an interview was filled in. Maternal blood pressure measurements were performed with a single standard device and after complete examination assurance was obtained that none of the participants had high blood pressure at the beginning of blood sampling. Then $5 \mathrm{cc}$ venous blood was taken from each one and daily samples at the end of sampling in a standard cold box were transported to the laboratory. Plasma of transported samples after preparation and separation by centrifugal device has been frozen at $70^{\circ} \mathrm{C}$ in specific freezers. All samples were coded before being frozen and till the end of second phase of sampling, remained intact in the laboratory. All subjects were followed for up to $48 \mathrm{~h}$ after delivery which asted approximately 9 months. Eventually in case of any suspicious sign of preeclampsia, by obtaining a complete history, measurement of blood pressure, checking the tests, performing clinical examinations, studying the patient's case and approval of preeclampsia by the physician, the 2 nd phase of blood sampling was performed. These people were categorized in the case group (preeclampsia group). Acceptable criteria for preeclampsia, according to the National High Blood Pressure Education Program include: blood pressure $\geq 90 / 140 \mathrm{~mm} \mathrm{Hg}$ on two occasions with an interval of $4 \mathrm{~h}$ measured with a single standard device by one of the researchers and proteinuria $\geq 300 \mathrm{mg}$ in $24 \mathrm{~h}$ surine that is $\geq+1$ in the urine-stick test. Among those who participated in the first turn of blood sampling those who did not develop signs of preeclampsia were considered as the controls. Thus, upon the occurrence of preeclampsia in one of the case group mothers from 5 patients of the study population which were matched with her in terms of maternal age and gestational age, the second stage of sampling was done and along with other samples of blood samples were stored in freezer. They were monitored until $48 \mathrm{~h}$ after delivery. If any of these 5 patients did not develop signs of preeclampsia, one of them was randomly selected and considered as a control case. And if an individual developed symptoms of preeclampsia risk was removed rom the control group and the other one was selected as a control case. After two stages of blood sampling plasma selenium levels were measured using atomic absorption spectrometry and then were compared and statistically analyzed. The data were analyzed using descriptive statistics, t-test and $\chi^{2}$ in the 0.5 level of significance.

In this study to determine the sample size PASS 2000 software was used and data collected were analyzed using the Statistical Package for the Social Sciences (SPSS11/5) and Stata 10.

\section{RESULTS AND DISCUSSION}

In this study, the case group (preeclamptic mothers) comprised $39(48.7 \%)$ and the other group comprised 40 
Res. J. Biol. Sci., 7 (2): 92-96, 2012

Table 1: Distribution of individuals in two groups based on levels of selenium (Phase I)

\begin{tabular}{lccccc}
\hline Groups & $\begin{array}{c}\text { No. of } \\
\text { individuals }\end{array}$ & $\begin{array}{c}\text { Low selenium } \\
<62.30(\%) \mathrm{n}\end{array}$ & $\begin{array}{c}\text { Medium selenium between } \\
62.2-74.4(\%) \mathrm{n}\end{array}$ & $\begin{array}{c}\text { High selenium } \\
>87.5(\%) \mathrm{n}\end{array}$ & \multicolumn{1}{c}{ Trend $\chi^{2}$} \\
\hline Preeclampsia & 38 & $16(42.1)$ & $14(36.8)$ & $8(21.1)$ & 7.3 \\
Control & 40 & $3(7.5)$ & $25(62.5)$ & $12(30)$ & $(\mathrm{p}=0.0060)$ \\
& & $12.75=\chi^{2}$ & & $\mathrm{p}=0.002$ & \\
\hline
\end{tabular}

Analysis for liner trend in proportions *(Epi software is used to calculate the analysis)

Table 2: Calculate the odds ratio in both low and average groups

\begin{tabular}{lcll}
\hline Groups & No. of people & Low selenium & Average selenium \\
\hline Preeclampsia & 35 & $16(42.1)$ & $14(36.8)$ \\
Control & 28 & $3(7.5)$ & $25(62.5)$ \\
& & OR $=9.52$ & $95 \%$ CL $(3.35 ; 38.46)$ \\
\hline
\end{tabular}

Table 3: Calculate the odds of both low and high

\begin{tabular}{lcll}
\hline Groups & No. of people & Low selenium (\%) $n$ & High selenium (\%) n \\
\hline Preeclampsia & 24 & $16(66.7)$ & $8(33.3)$ \\
Control & 15 & $3(20)$ & $12(80)$ \\
& & OR $=8$ & $95 \%$ CL $(1.4 ; 36.7)$ \\
\hline
\end{tabular}

$(51.3 \%)$ of mothers with normal blood pressure. There was no significant difference in terms of gestational age and type of eeding in the mean of two groups $(p<0.05)$. Percentiles of 25,50 and 75 based on the levels of selenium from the 1 st phase of two groups were determined (levels of selenium in low, medium, high), frequency distribution of two groups based on 3 levels of selenium were calculated and by using the $\chi^{2}$-test distribution of individuals in the two groups became apparent (Table 1).

Selenium levels in $42.1 \%$ of cases (preeclampsia) were low $(<62.2)$ while only $7.5 \%$ of the controls had low selenium levels. This difference was statistically significant $(\mathrm{p}<0.05)$. In fact, the majority of the patients in the case group had low selenium levels and the majority of the control group had average selenium levels. To calculate the odds ratio for the two groups of patients in low and medium levels of selenium are shown in Table 2. Chances of people with low selenium than those with average for preeclampsia are 9.52 (chances of people with low selenium were 9.52 greater than those with the average selenium levels).

The chances of people with low selenium in getting preeclampsia were 8 times more than than those with highlevels of selenium (Table 3 ).

To determine the best cutoff point the curve, ROC can be used. In ROC curve, the lower extensive area shows the more accurate identifying of those patients who are affected.

To determine the best cutoff point for early diagnosis of preeclampsia based and on the results, the 20,40 and 80 th percentiles were used. Then by using the cumulative frequency and logistic regression, predictive values were obtained. Sensitivity, specificity, positive and negative

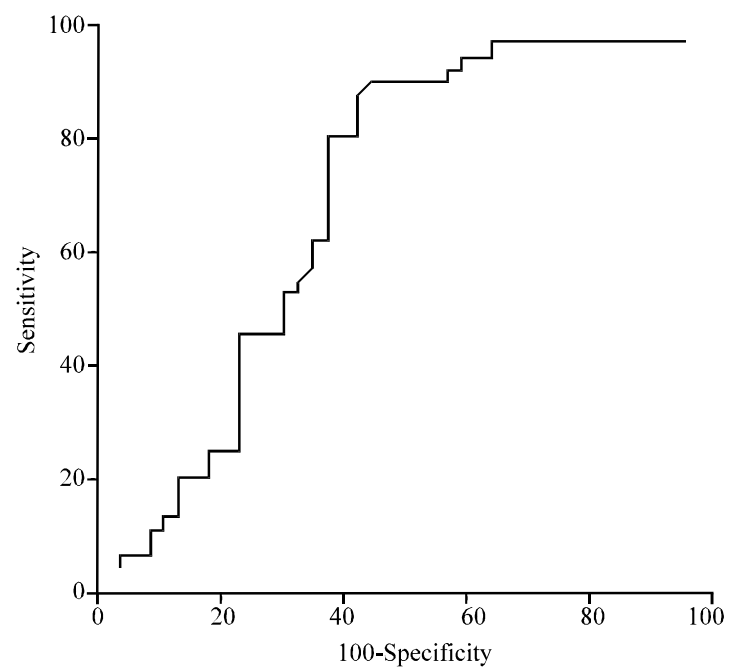

Fig. 1: Curve analysis of selenium to identify patients with extensive area under the curve that is equal to 0.71 and with reliability of $95 \%$ is between $0.6-0.81$

predictive values and likelihood ratio (chance) were calculated. By taking into account the interaction between the calculated values, the selenium levels of 60-70 showed the best cutoff point for prediction and early detection of preeclampsia. So a point between these two points was introduced as a cutoff point (Fig. 1). Using ROC curve analysis, the area under was 0.715 which was statistically significant and 0.5 shows the lowest area under the curve which represents $50 \%$ of diagnosed patients with this test. A large place under ROC demonstrates the accuracy of the test in diagnosing the patients.

Accordingly, the best cut point with sensitivity and specifity of $57.9,66.1$ and $90 \%$, respectively. The positive predictive value of 84.6 and negative predictive value of $69.2 \%$ and positive likelihood ratio of 79.5 and the negative likelihood ratio of 0.47 was estimated (Fig. 2).

Several studies have examined the relationship between blood selenium and preeclampsia. These have been designed primarily as case-control studies and measurements of serum levels have been done after the incidence of preeclampsia. One unique feature of the study was its design as a nested case, control study which measurements of blood plasma were performed before incidence of preeclampsia. Therefore allows possible prediction of preeclampsia occurrence with determination of blood selenium levels. 
Res. J. Biol. Sci., 7 (2): 92-96, 2012

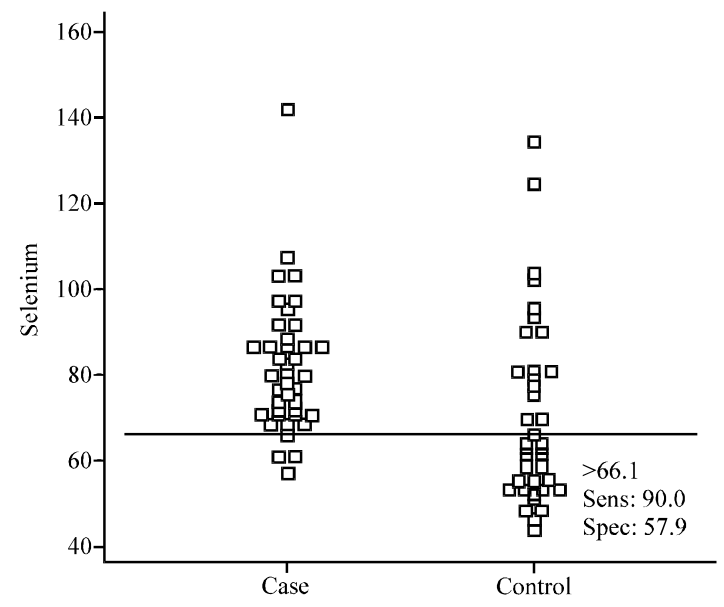

Fig. 2: The best cut-off point for selemnium in detection of healty and affected subject. The 66.1 point with $90 \%$ sensitivity and specificity of 57.9

The mean maternal plasma selenium levels of both case and control groups at 24-28 weeks of gestation showed that the average selenium levels in the case group was significantly lower than controls $(21.41 \pm 70.63$ vs. 15.54 \pm 82.03 ). This result was indirectly consistent with the results of some studies in other parts of the world (Kosanovic et al., 2002; Mahomed et al., 2000; Atamer et al., 2005; Mistry et al., 2008). Results of several studies suggested that selenium levels of amniotic fluid and cord blood in preeclamptic patients were significantly lower than non-affected (Dawson et al., 1999).

In the study of Rayman plasma selenium level in preeclamptic women was significantly less than levels of healthy pregnant women $(\mathrm{p}<0.000)$. The result was also indirectly consistent with the result of the study (Margaret et al., 2003).

According to the study of Gromadzinska et al. (1998) plasma selenium levels in preeclamptic group were $18 \%$ higher than the controls (Kosanovic et al., 2002). The result of this study was in contrast with the study.

In another study conducted by Rayman in England 19 preeclamptic pregnant women and 19 healthy pregnant women in third trimester were examined in selenium levels of serum. In that study, a statistically significant difference between the two groups in the blood selenium levels was not found (Rayman and Abou-Shakra, 1996). It seems that the population and the low sample size caused the statistical differences between the results with other similar studies.

The results of this study confirmed the destructive effect of selenium deficiency as an antioxidant in etiopathologic preeclampsia. Antioxidant selenoproteins can protect the endothelium by down-regulating cytokine-induced adhesion molecule expression and reducing inflammation (Mckenzie and Rafferty, 2001).
On the other hand, production of endothelial cells with sufficient selenium levels is the protection of cells against oxidative damages (Lewin et al., 2002; Dabbaghmanesh et al., 2006). Decrease in taking selenium before gestation reduces the concentration of Seleno-protein $\mathrm{p}$ in pregnancy and leads to the inability to protect against oxidative damages (Rayman and Abou-Shakra, 1996).

Since in this study, plasma levels of selenium at 24-28 weeks of pregnancy before the incidence of preeclampsia were measured, it was observed that in the 42.1 of those who ultimately were diagnosed preeclamptic had low selenium levels of $<62.2$ while only $7.5 \%$ of controls had this level. In this study, by using ROC curve the best cutoff point for prediction was obtained. Accordingly, the best cut point with sensitivity and specifity of 57.9 and $90 \%$, respectively was 66.1 . The positive predictive value of 84.6 and negative predictive value of $69.2 \%$ and positive likelihood ratio of 79.5 and the negative likelihood ratio of 0.47 was estimated.

In Rayman and Abou-Shakra (1996) study, the total level of antioxidants in 8-12 weeks was measured that had sensitivity about 33 and specificity of $95 \%$ in predicting pre-eclampsia (Dawson et al.,1999).

\section{CONCLUSION}

Lower plasma selenium levels in women destined to suffer from preeclampsia will confirm the destructive effect of selenium deficiency as an antioxidant inetiopathology of preeclampsia.

\section{ACKNOWLEDGEMENTS}

Researchers would like to thank the Vice Chancellor for research of Shiraz University of Medical Sciences and Endocrine and Metabolism Research Center of Shiraz University of Medical Sciences for their financial support. And also would like to thanks Seyed Reza Ghaemi for his guidance and support. Also, the researcher would like to thank Ms. Sara Medvari for improving the use of English in the manuscript. This manuscript is relevant to the thesis of Seyede Zahra Ghaemi with Project No. 89-5189.

\section{REFERENCES}

Atamer, Y., Y. Kocyigit, B. Yokus, A. Atamer and A.C. Erden, 2005. Lipid peroxidation antioxidant defens, status of trace metals and leptin levels in preeclampsia. Eur. J. Obstetric Gynecol. Reproductive Biol., 119: 60-66.

Cunningham, F.G. and J.W. Williams, 2005. Williams Obstetrics. 22th Edn., McGraw-Hill Prof. Med/Tech., UK., ISBN-13: 9780071413152, Pages: 1441. 
Dabbaghmanesh, M.H., A. Sadegholvaad, F. Ejtehadi and G. Omrani, 2006. Low serum selenium concentration as a possible factor for persistent goiter in Iranian school children. Biofactor, 28: 1-6.

Dawson, E.B., D.R. Evans and J. Nosovitch, 1999. Third trimester amniotic fluid metal levels associated with preeclampsia. Environ. Health J., 54: 412-415.

Gromadzinska, W., G. Wasowicz, D. Krasomsk, M. Broniarczyk, K. Andrijewski and K. Rydznski, 1998. Selenium level, thiobarbituric acid reactive substance concentrations and glutathione peroxidase activity labour. Analyst, 123: 35-40.

Hacker, S. and G. Moor, 2004. Essential of Obstetrics and Gynecology. 4th Edn., Saunders Punlisher, USA., pp: 199-201.

Kosanovic, M., M. Jokanovic, M. Jeuremovic, S. Dobric and D. Bokonjic, 2002. Maternal and fetal cadmium and selenium status in normotensive and hypertensive pregnancy. Biol. Trace Elem. Res., 89: 96-103.

Lewin, M.H., J.R. Arthur, R.A. Riemersma, F. Nicol and S.W. Walker et al., 2002. Selenium supplementation acting through the induction of the thioredoxin reductase and glutathione peroxidase protects the human endothelial cell line EAhy926 from damage by lipid hydroperoxides. Biochem. Biophys. Acta, 1593: 85-92.

Mahomed, K., M.A. Williams, G.B. Woelk, S. Modzamiri, S. Madzinc and I.B. King, 2000. Leukocyte selenium, zinc, copper concentration in preeclampsia and nornotensive pregnant women. Bio Trace Res. J., 75: 107-115.

Margaret, P., D. Rayman and B. Peter, 2003. Low selenium status is associated with the occurrence of the pregnancy disease preeclampsia in woman from the united kingdom. Am. J. Obstet. Gynecol., 189: 1343-1349.
Mckenzie, R.C. and T.S. Rafferty, 2001. Effect of selenium on immunity and ageing, molecular biology and role in health. Kluwer Academic Publisher, Dordrecht Netherlands, pp: 97-99.

Meads, C.A., J.S. Cnossen, S. Meher, A. Juarez-Garcia and G.T.D. Riet et al., 2008. Methods of prediction and prevention of preeclampsia: Systematic reviews of accuracy and effectiveness literature with economic modeling. Health Technol. Assess., 12: $107-178$.

Mehri, F., 2003. Nursing and Health of Mothers and Neonates. 1st Edn., Edition of Central Section of Jahad's University, Tehran.

Mistry, H.D., V. Wilson, M.M. Ramsay, M.E. Symonds and F.B. Pipkin, 2008. Reduced selenium concentrations and glutathione peroxidase activity in preeclamptic pregnancies. Hypertension, 52: 881-888.

Rayman, M.P. and F.R. Abou-Shakra, 1996. Comparison of selenium levels in preeclamptic and normal pregnancies. Biol. Trace Elem. Res., 55: 9-20.

Redman, C.W., G.P. Sacks and I.L. Sargent, 1999. Preeclampsia: An excessive maternal inflammatory response to pregnancy. Am. J. Obstet. Gynecol., 180: 499-506

Scott, J.R., R.S. Gibbs, B.Y.K. Krlan, A.F. Haney and T. Dastgerdi, 2005. Danforth's Obstetrics and Gynecology. 16th Edn., Tabib Publisher, Tehran, pp: 333-54.

Shils, M.E., M. Shike, A.C. Ross, B. Caballero and R.J. Cousings, 2006. Modern Nutrition in Health and Disease. 10th Edn., Lippincott Williams and Wilkins, A Wolters Klumer Company, pp: 280-281.

Zhang, C., M.A. Williams, I.B. King, E.E. Dashow and T.K. Sorensen, 2002. Vitamin $C$ and the risk of preeclampsia-results from dietary questionnaire and plasma assay. Epidemiology, 13: 409-416. 